\title{
LE GLOBE
}

Revue genevoise de géographie

\section{Récits de voyage : \\ Une géographie humaniste}

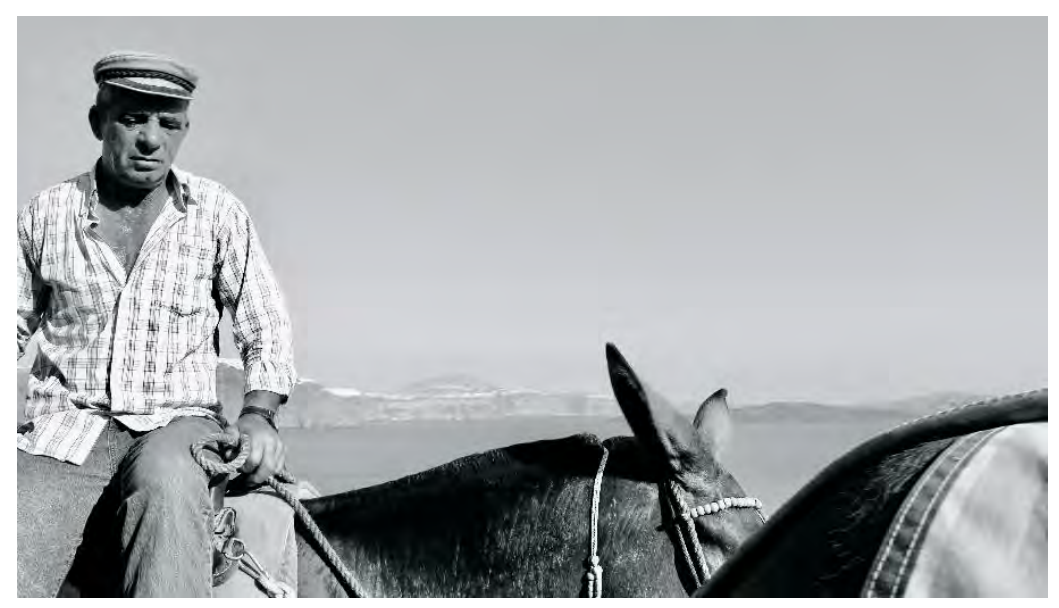

Tome $158-2018$ 
Le Globe est la revue annuelle de la Société de Géographie de Genève.

Il a été fondé en 1860 .

Publié avec le soutien de la Ville de Genève.

Comité éditorial :

Angelo Barampama, Ruggero Crivelli, Lionel Gauthier, Paul Guichonnet, Charles Hussy, Bertrand Lévy, Laurent Matthey, Claude Raffestin, Frédéric Tinguely, Jean-

Claude Vernex : Université de Genève.

Alain De l'Harpe, Philippe Dubois, Gianni Hochkofler, Philippe Martin, Christian Moser, Renato Scariati, Véronique Stein, René Zwahlen : Société de Géographie de Genève.

Elisabeth Bäschlin, Université de Berne

Rachel Bouvet, Université du Québec à Montréal

Sylvain Briens, Université de Paris-Sorbonne

Annabel Chanteraud, Université de Genève

Hans Elsasser, Université de Zurich

Franco Farinelli, Université de Bologne

Claudio Ferrata, GEA-Association des Géographes, Bellinzone, Tessin

Hervé Gumuchian, Université de Grenoble

Jean-Christophe Loubier, HES-SO Valais

Marina Marengo, Université de Sienne

René Georges Maury, Université de Naples

Jean-Luc Piveteau, Université de Fribourg

Jean-Bernard Racine, Université de Lausanne

François Taglioni, Université de Saint-Denis de la Réunion.

Rédacteur : Bertrand Lévy.

Coordinateurs du Tome 158 : Bertrand Lévy, Renato Scariati.

Lecteurs critiques du Tome 158 :

R. Bouvet, R. Crivelli, E. Galifi, G. Hochkofler, B. Lévy, J.-C. Loubier, L. Matthey, M. Marengo, R. Matos, C. Moser, R. Scariati, V. Stein, R. Zwahlen.

Tous les articles ont été soumis à lecture critique.

Les articles publiés dans Le Globe engagent la seule responsabilité de leurs auteurs Ils ne peuvent être reproduits sans autorisation des éditeurs.

Les propositions de publications sont à adresser au rédacteur :

Bertrand.Levy@unige.ch

Le Globe est une revue arbitrée par des pairs / a peer-reviewed journal.

Tirage : ca 450 ex.

Site internet : http://www.sgeo-ge.ch/le-globe

Le Globe est en ligne sur Persée : http://www.persee.fr/collection/globe

(C) Le Globe 2018

ISSN : 0398-3412 
LE GLOBE - TOME 158 -

RECITS DE VOYAGE : UNE GEOGRAPHIE HUMANISTE

SOMMAIRE

Littérature et géographie : dialogue autour du récit de voyage 5

Rachel Bouvet, Bertrand Lévy

Récit de voyage et expérience de l'espace : la Méditerranée écrite et vécue par Josep Piera

Alexandre Bataller

Le voyage en Italie de Pier Paolo Pasolini. Du reportage à

l'ichnographie : une paragéographie des seuils

41

Laurent Matthey

Le Voyage en France avec notes de Mariano Della Vedova :

le carnet de route et sa valorisation esthétique

Alba Dellavedova

Périples aquatiques - Impressions de voyages

Jean-Baptiste Bing

Retour à El Hierro

Jean-Michel Wissmer

La vie, la mort et les hommes. Moments choisis d'un voyage au Népal

Rémy Villemin

Il Pianto dei Balcani. Photo-poèmes de voyage à la recherche d'une histoire familiale

Sara Lonati

\section{Société de Géographie de Genève}

Bulletin de la Société de Géographie de Genève 


\title{
RECIT DE VOYAGE ET EXPERIENCE DE L'ESPACE : LA MEDITERRANEE ECRITE ET VECUE PAR JOSEP PIERA
}

\author{
Alexandre BATALLER \\ Dépt de didactique de la langue et de la littérature \\ Universitat de València
}

Résumé : La relation entre l'expérience du voyage et son écriture est analysée à partir d'échantillons de l'œuvre littéraire (prose autobiographique et poésie) de l'écrivain valencien Josep Piera. Trois concepts clés pour l'auteur sont abordés : le voyage, la Méditerranée et la littérature. La Méditerranée est conçue comme un monde proche et familier, en tant qu'espace géographique mythique, historique, culturel et vital. Les lieux visités (Grèce, Naples, Sicile, etc.) dialoguent avec l'espace et le temps personnels.

Mots-clés : Josep Piera ; Méditerranée ; Valence ; Littérature de voyage.

\begin{abstract}
Abstact : Based on some literary extracts (autobiographical prose and poetry) of the Valencian writer Josep Piera, the relationship between the experience of the journey and the writing that comes along as a result of it is analyzed. Three key concepts are discussed : the journey, the Mediterranean and literature. As we will see, the Mediterranean is understood here as a close and familiar world : a mythical, historical, cultural and vital geographical space. The places visited (Greece, Naples, Sicily, etc.) interact and produce a dialogue with the geographical space and the notion of human time.
\end{abstract}

Keywords : Josep Piera ; Mediterranean ; Valencia ; Travel literature.

Cet article aborde les récits de voyages de l'écrivain valencien Josep Piera (né à Beniopa, en 1947). Un auteur dont l'œuvre écrite en catalan se singularise par la combinaison de genres (poésie et prose) et de formes diverses de la "littérature du moi". En plus de la lecture de son œuvre, je connais personnellement l'auteur et il y a plus de vingt ans que j'ai mis en pratique des projets éducatifs avec des étudiants de lycée (Première et Terminale), fondés sur la lecture de ses livres de voyages. Ces projets incluaient autant le voyage qu'une visite à la maison de l'écrivain (fig. 1). Josep Piera habite près de Gandia, à La Drova, une vallée de montagne qui fait face à la Méditerranée, un paysage qui dialogue avec les 
déplacements de l'auteur et où il revient toujours, comme un Ulysse contemporain.

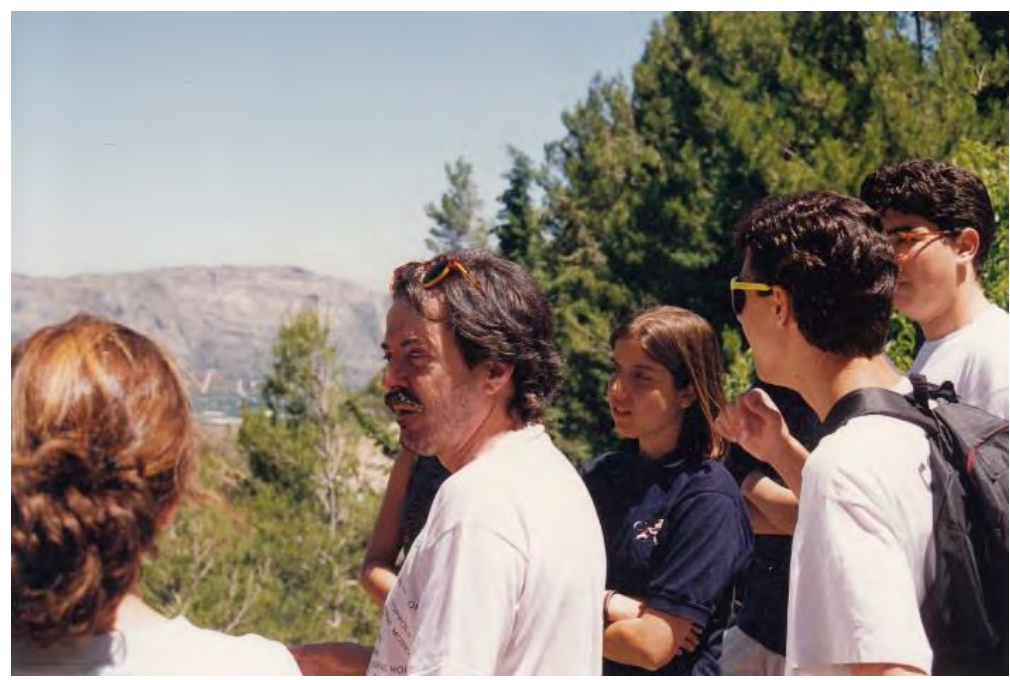

Fig. 1 : L'écrivain Josep Piera explique son paysage aux étudiants de lycée (juin 1995). Au fond, la Valldigna, une vallée qui regarde la Méditerranée (Photo : A. Bataller)

\section{Josep Piera et la littérature de voyage}

Josep Piera est un des rares auteurs de la littérature catalane contemporaine - à l'exception de la figure notable de Josep Pla - qui se soit intéressé à la littérature de voyage, et concrètement au monde méditerranéen (pour le cas du voyage en Italie, voir Bataller, 2017). Parmi ses œuvres, j'ai sélectionné pour cet article neuf d'entre elles qui ont été publiées dans une période couvrant trente-quatre ans. Sauf indication contraire, les fragments des ouvrages cités et les écrits critiques sont présentés et traduits en français par Teresa Lajoinie.

- El cingle verd (1982) [La falaise verte] à propos de La Drova et de la Grèce.

- Estiu Grec (1985) [Eté grec] aussi à propos de la Grèce.

- Un bellíssim cadàver barroc (1987) [Un très beau cadavre baroque] à propos de Naples.

LE GLOBE - TOME 158 - 2018 
- Ací s'acaba tot (1993) [Ici tout s'achève] à propos de la Sicile.

- Seduccions de Marràqueix (1996) [Séductions de Marrakech].

- A Jerusalem (2005) [A Jerusalem].

- Grèce (2007), la seule de ses œuvres traduite en français.

- El temps tobat (2013) [Le temps trouvé], livre de poésie où la Méditerranée et le voyage sont protagonistes.

- Europa al vol (2016) [L'Europe au vol], son dernier livre sur le thème de la Méditerranée.

\section{Le concept de voyage}

Piera explique qu'habituellement il voyage seul et que la littérature s'avère être l'ami-amant nécessaire pour que le voyage touche son plus haut degré d'intensité : "Ce ne sont pas des voyages à travers des mondes exotiques mais à travers des mondes qui sont proches, familiers. La Méditerranée, précisément, constitue le monde le plus proche et familier, celui qui nous appartient le plus" (Mora, 1990:68).

Le voyage de Piera est une expérience de changement et d'étrangement, de fuite et de recherche de soi par le biais du contact avec une réalité nouvelle, mais d'une altérité connue, familière et proche (Siviero, 2012).

Comme dans tout voyage, on parle du mouvement dialectique de fuite et de retour : la première partie implique le désir du voyageur de laisser son environnement, comme dans un processus d'initiation; le retour implique une vita nuova, un enrichissement (Scarpi, 1992). A la fin de Seduccions de Marràqueix, le pèlerin étant revenu chez lui, nous pouvons lire : "Rien n'est comme je l'avais laissé lorsque je suis parti en voyage, tout est différent sans que rien ne soit changé, tout est nouveau sans que je ne le sente étrange. Ni les personnes sont différentes, ni les objets, ni la maison, ni cette ancienne vallée où j'habite; c'est moi qui regarde avec d'autres yeux et je découvre ce qui m'était caché avant. C'est moi qui maintenant est un autre. J'observe les montagnes que j'ai devant, avec des champs d'oliviers et d'amandiers que plus personne ne travaille et des marges de pierres grises qui tombent, avec des lentisques et des ajoncs plus haut ; son nom, Aldaia, en arabe veut dire jardin" (Piera, 1996:205).

Le voyage n'est jamais pour lui une fuite: "Et je ne veux fuir de nulle part, moi je voyage tout simplement pour sentir le temps vivant - tous les temps -, pour me sentir vivant dans le temps ; pour ne pas perdre de vue ce que j'ai près de moi, pour ne pas laisser derrière et dans l'oubli qui je 
suis, mais pour avoir près de moi ce qui est loin, me sachant quelqu'un qui devient plus lui pendant qu'il voyage, car le voyage lui permet de laisser séduire sa volonté et son âme par ce qui l'entoure, lui permet la jouissance permanente de chaque instant, des rêves même" (Piera, 1996:12).

Le voyage constitue une métaphore de la vie et elle est quadruple : le voyage dans le temps (voyage au passé), le voyage dans l'espace (voyage en Méditerranée), le voyage dans la mémoire (les souvenirs du voyage) et le voyage dans la littérature (le livre de voyages) (Climent, 1996 ; Maestre, 2001:253). Dans le voyage, Piera reconnaît les principaux axes de sa conception littéraire : le présent hasardeux (loin de la monotonie de la quotidienneté), le paysage, le proche, l'évocation libre du souvenir, et la fiction de ceux qui le précédèrent (Esteve, 2012:64).

Le moi de l'auteur nous fait penser à un voyageur romantique qui cherche dans la Méditerranée la matérialisation de ses mythes culturels : "Même si l'auteur veut s'éloigner de l'image stéréotypée de l'écrivain romantique, dans ses œuvres il est possible d'observer des traits qui le rapprochent de ce modèle de voyageur littéraire" (Maestre, 2001:269).

Si Lévi-Strauss avait prédit au milieu du $\mathrm{XX}^{\mathrm{e}}$ siècle la fin du voyage, car dans le monde il n'existait plus de territoires n'ayant pas été explorés, les récits de voyage de Josep Piera montrent la recherche dans les pays méditerranéens d'une humanité à l'état primaire, avec le topos du "paradis perdu", tant dans la civilisation que dans l'enfance.

Selon Maestre (2001:285), avec ses livres de voyages, Piera entreprend la reconstruction de l'espace mythique, historique, culturel et vital de la Méditerranée. Pour l'auteur, cet espace symbolise l'origine de la civilisation. Voyager à travers les pays qui le forment signifie découvrir les racines de sa culture puis le début de ses propres racines : l'enfance. Les origines de l'histoire étant, ainsi, l'équivalent des origines de la vie.

Le voyage en avant de ce qu'il trouve n'est autre que le voyage en arrière de ce qu'il retrouve. Nous lisons : "A Marrakech, ce que je voyais me transportait aux paysages de mon enfance, à d'autres printemps perdus" (Piera, 1996:205). Il y va pour se perdre, pour se retrouver, et il y découvre son monde évoqué. Il s'aperçoit que, loin de chez lui, il est cependant très près; qu'il a voyagé dans le même lieu dont il était sorti et duquel il provient. Le paysage naturel et humain est partagé, et tout se remplit de la sagesse et la sensualité qu'il peut aussi trouver là d'où il vient. Par exemple, le Maroc qu'il visite, le Maroc qu'il vit, le ramène à son enfance, et ceci

LE GLOBE - TOME 158 - 2018 
fait que ce qui dans un premier temps était un livre de voyages vers l'extérieur, devient une aventure intime, une incursion en lui-même (Forcano, 2012).

Ce mécanisme pendulaire est essentiel pour comprendre les récits de voyages de Piera. Un voyage de la maison vers un monde qui rappelle la maison, un voyage du monde à une maison qui rappelle le monde. Tout ce que Piera voit et ce qu'il apprend à travers le monde méditerranéen lui rappelle tôt ou tard sa maison. Dans ce qui est exotique il retrouve ce qui est familier, de la même manière que dans le familier il trouve l'exotique (Sòria, 2008).

Nous proposons ensuite de considérer, à titre d'exemple, un passage concret d'un récit de voyage. Le livre $A$ Jérusalem est un voyage vers la capitale d'Israël, lors d'une croisière qui devait l'amener jusqu'à l'autre bout de sa Méditerranée. L'un des passages décrit le Tombeau du Jardin, un parc situé à l'extérieur des remparts de la vielle cité de Jérusalem où les diverses confessions chrétiennes protestantes croient que se trouve le vrai lieu, la localisation réelle du Golgotha et du sépulcre de Jésus de Nazareth. Piera se promène dans ce jardin tranquillement et pense aux souvenirs du passé, et il nous transporte tout à coup dans le jardin de sa propre maison, dans son paysage, dans son village de la Drova, la vieille vallée où il vit son Orient personnel, et où il a devant lui la chaîne de montagnes appelée Aldaia (fig. 2). Piera, à Jérusalem, au Tombeau du Jardin de Jésus de Nazareth, à un emplacement considéré comme sacré pour tant de pèlerins, laisse sa pensée s'envoler vers la Drova et, au lieu de se laisser posséder par la sainteté et la transcendance du lieu, ce qu'il fait est mythifier, sanctifier et vénérer son jardin particulier à la Drova, où il habite, d'où il est, le lieu auquel il appartient réellement (Forcano, 2012).

\section{La Méditerranée de Josep Piera}

Les espaces de ses voyages comprennent, fondamentalement, la Grèce et les îles de la mer Egée ; Naples, un paradoxe entre la vie et la mort, le vitalisme et la corruption; la Sicile, où il expérimente l'idée de la fin lorsqu'il se souvient du voyage à partir d'un long séjour hospitalier; Marrakech, l'oasis à l'entrée du désert; et Jérusalem, un espace de confrontation, l'utopie entre le Ciel et le Paradis. 


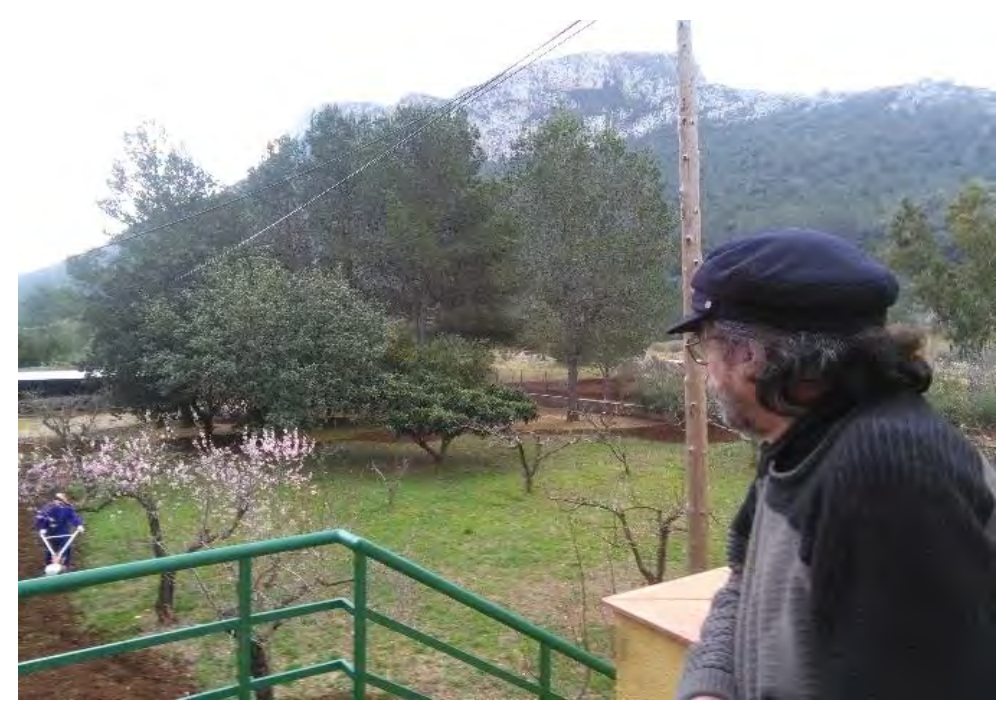

Fig. 2 : Josep Piera dans sa maison à la Drova (février 2017). Au fond, la Falaise Verte, dans la chaîne de montagnes appelée Aldaia. En dessous, un amandier en fleurs (Photo : A. Bataller)

L'écrivain affirme que son premier voyage en Grèce signifie tant découvrir le monde des classiques que sa propre enfance ; il explique que se promener à travers les îles grecques, c'est se promener à travers un paysage de fiction dans lequel il peut contempler son enfance rurale, d'oliviers, de vignes et de personnes endeuillées dans les rues (Mora, 1990:35-36).

Il est important de souligner que les trois traditions de l'espace personnel de Josep Piera sont l'héritage gréco-latin, le provençal et ses troubadours, puis l'arabe de l'Al-Andalus (Pont, 2008:59). Le monde méditerranéen est une synthèse de l'Orient et de l'Occident et l'auteur se considère comme un enfant de la Méditerranée (Mora, 1990:38). Ceci est indiqué dans un entretien : "Je suis un fils de la Méditerranée, je vais me chercher. Mes origines sont la Grèce, mais c'est aussi Al-Andalus et c'est aussi la culture catalane. Et moi, tout cela, je le redécouvre en Méditerranée, qui est mon espace naturel, même national, culturel" (Lluró, 1990:38).

LE GLOBE - TOME 158 - 2018 
Ses œuvres représentent une analyse et une construction de sa propre identité, individuelle et collective. En conséquence, le "moi" laisse souvent la place au "nous". Les Valenciens intégrés dans une communauté catalane qui, à son tour, s'intègre dans la culture méditerranéenne (Maestre, 2001:270). Les Méditerranéens étant, en plus, les "Européens du sud" (Piera, 1993:109). Il écrit: "Comment peut-on douter de l'existence d'une condition méditerranéenne multiple? Tant au niveau du territoire, que dans le plan historique et culturel, les Valenciens, d'un point de vue universel, sont liés à la Méditerranée. Si, à une certaine époque, nous avons eu quelque rôle de protagoniste en Europe, c'est dans ce cadre donné. Ce que nous nous sentons, nous, héritiers d'une culture et mentalité catalane, nous, Valenciens, fils de cette histoire, c'est dans cette réalité que le monde nous donne une place, où le temps parle pour nous (...). Je parle de la conscience historique à partir de l'amour pour le passé collectif, à partir du maintien en vigueur de la collectivité, à partir du maintien en vigueur d'une tradition culturelle. La Grèce sur ce point est un magnifique modèle" (Piera, 1985:88).

A ce propos, l'écrivain voyageur explique que : "La Grèce n'est pas seulement l'enfance de l'Occident, mais aussi notre propre enfance personnelle en tant qu'individus" (Piera, 1982:141). En Grèce il redécouvre le paysage de son enfance et la culture de l'Europe du sud. Une manière de sobriété liée à la nature, à la jouissance des choses simples et essentielles: "En quoi la Grèce me fascine? L'émotion d'être dans les lieux des divinités, en constant dialogue avec personne, spectateur des passés et des futurs, installé dans l'instant. Moi, enfant d'un pays ignoré, né au bord de cette mer ancienne, héritier de milliers de défaites, chair d'oubli et de silences, grâce à la Grèce je récupère la paix et la conscience d'être qui je suis, voyageur de la vie" (Piera, 1985:12).

Ses récits sont truffés de comparaisons par rapport à son pays, à l'unité du paysage, des mœurs, de l'histoire, des ressemblances gastronomiques et linguistiques: "Petit-enfant de paysans comme je suis, la ruralité grecque me fait sentir chez moi et je retrouve, ainsi, d'où je viens, qui je suis. Voyager à travers la Grèce, de cette manière-là, n'est pas seulement se déplacer à travers l'espace géographique d'une nation concrète, mais aussi entrer dans le temps et les souvenirs intimes, tant collectivement qu'individuellement" (Piera, 1985:142).

LE GLOBE - TOME 158 - 2018 
C'est pour cela qu'à Ios, dans la mer Egée, en expliquant pourquoi ce qui l'intéresse ou ce qu'il préfère de la Grèce est de chercher tout ce qui lui permettrait de vivre et de sentir la vie, il dit : "(...) c'est de cette manière que les lieux, les objets, les personnes nous offrent une partie d'euxmêmes, un morceau de leur être (...) et nous pouvons les aimer. Tout possède un élément de beauté, un détail aimable, un instant parfait. Le trouver et le sentir (...) c'est ce que je recherche" (Piera, 1985).

Le voyage à Naples se révèle comme une forme de connaissance personnelle. Comme Stendhal, qui se propose de saisir l'esprit de Rome et de s'y identifier, dans ses Promenades dans Rome (de 1828 et 1829), Josep Piera veut découvrir Naples, tentant l'effort de raisonner à travers la ville une partie jusqu'alors ignorée de lui-même (Saladrigas, 1988). Il observe aussi les connexions entre les villes de Naples et de Valence. L'auteur explique plus en détail, dans un entretien, que "venant d'une ville où le passé a été enterré ou aboli consciemment, Naples est impressionnant. A Valence l'on vit la nostalgie d'un passé effacé, inexistant; à Naples, l'omniprésence d'un passé magnifique d'une ville qui n'a pas de futur" (Bertomeu, 1988:58). Le parallélisme nord-sud se transpose à sa propre réalité : Valence. De la puissance économique, politique et culturelle pendant le Moyen Age, nous sommes passés, à partir de notre inclusion dans un état plus grand, à une décadence de laquelle nous ne sommes toujours pas sortis. Il écrit alors : "Souvent, ici, lorsque je dois expliquer d'où je viens, j'ai l'habitude de dire : nous, les Valenciens nous sommes comme les Catalans du sud, comme les Napolitains du sud ; capito? Nous faisons tous partie de ce sud européen, méditerranéen, rural et ancien, vital et misérable, détruit par les autochtones. Et ils me comprennent" (Piera, 1987:74).

Le titre de son livre napolitain est assez explicite à cet égard: "Oui, Naples c'est cela: un beau cadavre plein de vers humains qui se multiplient au fur et à mesure que la corruption de la mort avance. Un splendide cadavre baroque" (Piera, 1987:43).

Ces observations sont présentes aussi dans son voyage en Sicile qui, pour l'auteur, représente le symbole et la décadence du monde méditerranéen. La Sicile est une île littéraire, un territoire qui existe dans les livres, la création des écrivains séduits par l'image créée par d'autres écrivains : "La Sicile, pour moi, est la réalité du mythe déchu. C'est la synthèse historique et la présence la plus digne, la plus sublime et le temps

LE GLOBE - TOME 158 - 2018 
dégradé. C'est, en outre, un monde de souvenirs, un monde littéraire" (Piera, 1993:130). L'écrivain considère que Catane, Naples et Valence sont toutes trois des villes contradictoires, vitalistes, tragicomiques, folles, théâtrales, lumineuses et tourmentées, avec un passé brillant et submergées dans un même oubli.

\section{L'écriture de Josep Piera}

Josep Piera écrit ses livres de voyages dans le présent pour narrer des événements du passé. C'est la mémoire qui supprime, augmente et réorganise l'expérience du passé, à partir de son cahier de notes du voyage. En conséquence, le récit de souvenirs est prédominant, un récit conçu comme la narration de vécus personnels du passé.

Ací s'acaba tot (1993), par exemple, se construit à partir des souvenirs d'un moi, d'un "voyageur qui courrait éperdu à travers la Sicile" (Piera, 1993:9), qui revit à travers la mémoire l'expérience de ce voyage.

De son côté, Un bellíssim cadàver barroc (1987) est un journal intime d'un voyageur qui habite et qui vagabonde à Naples et alentours et raconte jour par jour son expérience. A cette occasion, il se produit un certain décalage temporel entre ce qui s'est passé et ce qui est retenu dans le récit ; il s'agit d'une "rétrospection de faible portée" (Rousset, 1983:435).

Le voyage important est celui des mots : "Oui, celui-ci est un voyage de mots, me dis-je dans le train direction Taormine. Oui, celui-ci est le vrai voyage, quand les choses se sentent être plus réelles grâce à l'écriture, une écriture lente et mesurée qui reconstruit ce temps-là, maintenant passé, mais intensément présent dans la mémoire, présent et vif grâce à ces notes pressées sans lesquelles il me serait plus difficile de me situer à nouveau dans les lieux et dans les détails de ce voyage. C'est grâce à la réalité de ces mots récupérés que je peux aller loin, à Taormine encore une fois. Dans le voyage vécu à partir du souvenir, dans le voyage mental des mots, dans le vrai voyage" (Piera, 1993:108). Il le dit également à un autre moment : "Alors, dans le journal intime, j'avais noté : Je me fais de la compagnie avec les mots. Avec eux je vis à nouveau le meilleur de chaque jour et je garde pour moi les arômes, les images et les saveurs préférés. J'essaye, aussi, d'oublier que je vais seul, que je mange seul, que je dors seul" (Piera, 1993:29).

Deux types de citations sont incorporées dans le texte du livre de voyages : celles qui ont pour but de prouver l'authenticité de l'expérience

LE GLOBE - TOME 158 - 2018 
(conversations, inclusion de documents, citations du journal intime ou notes); et celles qui contiennent une marque de culture et invoquent un autre type de référentialité, tradition ou intertextualité (Grudzinska, 198990:231).

Par exemple, dans son voyage en Sicile l'auteur cherche à rencontrer l'écrivain Gesualdo Bufalino et le décrit comme étant: "Fragile, mince, élégant, Gesualdo Bufalino m'avait reçu pour prendre un café. Dans un salon plein de livres et deux grandes fenêtres dans le coin. Son aspect était celui d'un petit homme de village, extrêmement poli. Né à Comiso, il fut professeur jusqu'à la retraite (...)" (Piera, 1993:88-89). Mais il profite aussi de lui donner une voix propre la transposant au texte sous le style direct : " Comiso est un village de la Sicile amère, comme de nombreux autres (...). Nous voudrions avoir le temps de prendre soin des personnes que l'on aime, de s'occuper des livres, du cinéma, pour se balader... Avezvous vu Nuovo Cinema Paradiso ? Ne manquez pas d'y aller" (Piera, 1993:88-89). Piera combine même la narration et la description : "Lorsque l'autobus qui me ramène à Raguse laisse en arrière la plaine agricole de Comiso et monte en haut de la colline, au milieu d'amandiers invisibles et d'oliviers fantomatiques, en bas, la plaine pleine de lumières semble une foire" (Piera, 1993:88-89).

Dans ce sens, nous pouvons considérer Séductions de Marrakech comme une comparaison entre les livres qu'il lit - Ibn Khafaja, Paul Bowles, Juan Goytisolo, Elias Canetti, etc. - , les choses qu'il voit et les conversations qu'il entretient avec les gens du lieu.

Concrètement, Piera cherche une expérience personnelle de l'espace : "Je tente une relation intime avec l'environnement. La ville ne doit pas être uniquement l'espace-décor d'une histoire. Elle doit être l'amante ou la compagne dans ce vivre solitaire actuel" (Piera, 1987:141). Une expérience comme fondement de l'écriture : "(...) j'aime, simplement, me laisser porter par le temps et par l'espace, n'attendre autre chose que quelques sensations ou images éphémères qui arrivent à surprendre la rétine et nous font être attentifs à tout, conscients de la propre vie." (Piera, 1985:130).

Si l'espace sépare les choses et les personnes, le temps, le récit, les réunit. En conséquence, le discours se remplit d'histoires réelles ou racontées, documentées ou faites de rumeur; avec des images, des comparaisons, des liens et des contre-points. Les souvenirs, dit Piera, sont

LE GLOBE - TOME 158 - 2018 
la colonne vertébrale de la vie. Ce sont eux, ceux qui nous configurent (Calafat, 2008). Le mot permet, enfin, de "rendre la vie éternelle" (Ferrer, 2008:38).

\section{Trois textes de Josep Piera}

Dans le but d'exemplifier ce qui vient d'être dit, nous fournissons un ensemble de textes de Josep Piera dont les récitations peuvent être écoutées à travers des liens qui sont indiqués en note.

\section{Texte 1}

L'Ode à Santorin ${ }^{1}$ est un poème dédié à cette île, émergée de l'eau comme un mystère des dieux, et qui apparaissait déjà dans son premier livre en prose et dont l'enregistrement en catalan, langue de l'original, peut être entendu sur le site de l'Institut Ramon Llull².

\section{Ode à Santorin}

La sombre ardeur du plaisir que je cherche sur cette mer de lumière et d'espérances, plaisir d'être cet enfant d'étoiles qui un jour s'est senti rêve d'ange, m'a conduit ici, comme un navire à la dérive, jusqu'à ton port de cendres moulues, lune de l'Egée bleue, feu endormi comme l'oiseau qui renaît en s'immolant.

Ile du ciel, née de la flamme, stérile en flore, fertile en hécatombes, je te désirais tant, je t'aimais tant, qu'avant de posséder tes abîmes je te voulais déjà telle que tu es, fille de l'aube avec tes vignes accrochées à la vie, à-pics taillés à la hache, tes plages de froide lave, où l'homme, comme les rochers, chante et pleure.

(Durazzo, 2005).

\section{Texte 2}

Nous continuons avec le poème De bord à bord ${ }^{3}$ qui permet d'établir un dialogue entre les deux bords de la Méditerranée. En premier lieu, il 
rend possible le voyage géographique dans les lieux, aux endroits du passé. La ville portuaire de Dènia, sur la côte valencienne, évoque celle de Tipasa, située sur la côte d'Alger. Il permet le voyage à travers la culture (avec une référence explicite à Albert Camus). Tipasa, l'antique port romain posé à 80 kilomètres à l'ouest d'Alger, fait partie de la géographie sentimentale d'Albert Camus, qui s'est trouvé ici en juillet 1937 avec la jeune Oranaise Christiane Galindo. Il y a une stèle en mémoire des Noces à Tipaza d'Albert Camus, essai écrit en 1939, sur laquelle est inscrit un extrait : "Je comprends ici ce qu'on appelle gloire : le droit d'aimer sans mesure. Il n'y a qu'un seul amour dans ce monde" (Camus, 1945). Il permet le voyage vers l'histoire personnelle (l'expérience de la passion de jeunesse près de la mer tant chez Camus que chez Piera). Puis, il permet, en définitive, l'union entre les deux bords de la Méditerranée.

De bord à bord

\section{A Albert Camus}

Tipasa était un cimetière de ruines au bord de la mer, avec un monolithe en pierre rongé par le sel, où il était écrit :

Je comprends ici ce qu'on appelle gloire :

le droit d'aimer sans mesure.

Devant tant de bleu je sentais l'air propre, la lumière de sel, les ondes se défaisant en mots, ton corps désiré

à toucher, tout et loin loin et tout. A l'autre bord, tu m'attendais avec le soleil et le sel et le délit et le délire à la peau. Tipasa était Dènia lorsque toi et moi à vingt ans nous nous frottions ensemble.

A Dènia, comme je sens maintenant à Tipasa, j'ai appris que - le plaisir évanoui la souffrance se présente d'un coup.

La lumière aux yeux. Odeur de sel du bord. De poisson frais à la braise. D'ombre plaisante au milieu de fumées vertes et gorgées de thé, je sentais Dènia rencontrée sans frissons.

\section{LE GLOBE - TOME 158 - 2018}


Tipasa : sarcophages avec des iris bleus.

(Piera, 2013:28-29 ; traduction : T. Lajoinie)

\section{Texte 3}

Finalement, nous présentons un fragment du texte La Méditerranée $e^{4}$ que l'on peut considérer comme une déclaration de principes à propos de la condition méditerranéenne de l'auteur, avec des références littéraires personnelles. L'extrait appartient à son livre le plus récent, et il y inclut des allusions aux réfugiés et à ceux qui risquent leur vie pour traverser d'un bord à l'autre la Méditerranée.

\section{La Méditerranée}

Je me suis senti méditerranéen aux marchés de Naples, devant le portail du Castel Nuovo d'Alphonse le Magnanime, en prenant une pizza à Port'Alba avec Virgile et Dante, au café Gambrinus bavardant amicalement, à Ravello en rêvant des dieux...

Je me suis senti méditerranéen aux ports de Gênes et de Marseille, en voyant les navires de tous les temps partir vers des mondes vieux et nouveaux, vers le lointain et vers nulle part, au milieu des parfums de lavande, de sel, de savon et, hélas ! des lauriers lyriques de Mistral. Au port de Sète en mangeant des fruits de mer au milieu du revoler des mouettes et en récitant les vers bleus de Valéry.

[...] Maintenant que cette mer à tous est devenue tragique, maintenant qu'elle est traversée par des fugitifs de la mort qui échappent aux guerres lointaines en cherchant la vie, la paix et la liberté, maintenant que les plages de Lampédouse, de Ceuta, de Lesbos ou de Kos apparaissent avec des cadavres innocents ou avec des gilets de sauvetage laissés comme des fleurs aux tombeaux... Maintenant que de la Méditerranée on dit qu'elle est la mer des morts. Maintenant que la Méditerranée est appelée la mer des morts, maintenant que dans le deuil et dans la plainte comme avant dans le désir et dans la joie, je me suis senti méditerranéen.

Parce que ce n'est pas que je sois méditerranéen, c'est que je me sens l'être de cœur, de tête, de vie, de parole et de voix."

(Piera, 2016:11-16 ; traduction : T. Lajoinie). 


\section{Conclusion}

Josep Piera est un écrivain qui se retrouve dans le voyage et la littérature. Le paysage de la Drova (Gandia, Valence) où se trouve sa maison, point de départ du voyage et de l'écriture, dialogue avec le reste des espaces. L'expérience personnelle de l'espace méditerranéen et l'écriture autobiographique sont comprises comme des formes de connaissance. Les mots de l'écrivain sicilien Vincenzo Consolo, reproduits par l'écrivain valencien dans un texte programmatique sont une synthèse de la relation entre l'expérience du voyage et la révélation de la poésie: "la narration poétique (non pas le roman) est la restitution impraticable d'une expérience, et particulièrement de l'expérience d'un voyage, selon la définition de Walter Benjamin. Et cette narration est à la fois chant et enchantement, révélation et occultation, vérité et mensonge, muse et sirène, mémoire et oubli. C'est, si on le veut, la re-création d'une autre vérité, la vérité de la poésie" (Piera, 2009:22-23 ; à partir de Consolo, Nicolao, 1999).

\section{Bibliographie}

Bataller A., 2017, "Tres miradas del viaje a Italia desde la literatura catalana: Josep Pla, Vicent Andrés Estellés y Josep Piera", in : Anderson L., Marengo M., Micali S., Schoysman A. (eds), Stranieri di carta, stranieri di voce, Roma, Artemide, pp. 169-182.

Bertomeu C., 1988, "Josep Piera, poeta en prosa", El Temps, No 185, pp. 57-59.

Calafat F., 2008, "La vida com la literatura", L'Aiguadolç, No 35, pp. 65-80.

Camus A., 1945, Noces à Tipasa, Paris, Presses de l'Impr. Curial-Archereau.

Climent E., 1996, "Marràqueix, retorn al paradís perdut", El Temps, № 651, pp. 66-68.

Consolo V., Nicolao M., 1999, Il viaggio di Odisseo, Milano, Passaggi Bompiani.

Durazzo F.-M. (ed.), 2005, 48 poètes pour le XXI siècle, anthologie de la poésie catalane, Trois-Rivières (Québec), Ecrits des Forges.

Esteve A., 2012, "'La memòria, com el desig, s'esborra a poc a poc i es fa ficció'. La prosa autobiogràfica de Josep Piera", in : Josep Piera. Actes de la II Jornada sobre els Escriptors Valencians Actuals, València, Publicacions de l'Acadèmia Valenciana de la Llengua, pp. 59-68.

LE GLOBE - TOME 158 - 2018 
Ferrer A., 2008, "La Grècia possible de Josep Piera", L'Aiguadolç, № 35, pp. 23 38.

Forcano M., 2012, "L'Orient de Josep Piera", in : Josep Piera. Actes de la II Jornada sobre els Escriptors Valencians Actuals, València, Publicacions de l'Acadèmia Valenciana de la Llengua, pp. 69-76.

Grudzinska I., 1989-90, "Stendhal, Travel Writing, and Plagiarism", Nineteenthcentury French Studies, Vol. XVIII, No 1-2, pp. 231-235.

Lluró J. M., 1990, "Josep Piera: per fugir de la derrota", Lletra de canvi, № 28, pp. 34-39.

Maestre A., 2001, "La narrativa de viatges de Josep Piera: la identitat mediterrània", in: Miscellània Giuseppe Tavani, Barcelona, Abadia de Montserrat, Vol. 2, pp. 250-286.

Mora I., 1990, "Josep Piera: La literatura o la vida", El Temps, 30-4-90, pp. 6669.

Piera J., 1985, L'estiu grec, Barcelona, Destino.

Piera J., 1987, Un bellissim cadàver barroc, Barcelona, Edicions 62.

Piera J., 1993, Ací s'acaba tot, Barcelona, Edicions 62.

Piera J., 1996, Seduccions de Marràqueix, Barcelona, Edicions 62.

Piera J., 2005, A Jerusalem, Barcelona, Edicions 62.

Piera J., 2009, Grèce, València, Institució Alfons El Magnànim.

Piera J., 2013, El temps trobat, Alzira, Bromera.

Piera J., 2016, Europa al vol, Catarroja, Perifèric.

Pont J., 2008, "Una genealogia poètica i cultural (la poesia andalusina en l'obra de Josep Piera)", L'Aiguadolç, No 35, pp. 45-64.

Rousset J., 1983, "Le journal intime, texte sans destinataire ?", Poétique, № 56, pp. 435-443.

Saladrigas R., 1988, "La aventura de medir el tiempo", La Vanguardia, 14-11988, p. 37.

Scarpi P., 1992, La fuga e il ritorno. Storia e mitologia del viaggio, Venezia, Marsilio. 
Siviero D., 2012, "Itàlia i la Mediterrània en l'obra de Josep Piera. Una aproximació a 'Un bellíssim cadàver barroc' i 'Ací s'acaba tot"', in : Josep Piera. Actes de la II Jornada sobre els Escriptors Valencians Actuals, València, Publicacions de l'Acadèmia Valenciana de la Llengua, pp. 77-88.

Sòria E., 2008, "Josep Piera o la creació del temps", L'Espill, № 30, pp. 138-145.

\section{Notes}

1. https://mmedia.uv.es/buildhtml/51088

2. http://poetarium.1lull.cat/poetarium/detall.cfm/ID/26841/FRA/joseppiera.html

3. https://mmedia.uv.es/buildhtml/51087

4. https://mmedia.uv.es/buildhtml/51089

LE GLOBE - TOME 158 - 2018 